\title{
Iris: an Extensible Application for Building and Analyzing Spectral Energy Distributions
}

\author{
Omar Laurino $^{\mathrm{a}, *}$, Jamie Budynkiewicz ${ }^{\mathrm{a}}$, Raffaele D'Abrusco ${ }^{\mathrm{a}}$, Nina Bonaventura ${ }^{\mathrm{a}, 1}$, Ivo Busko ${ }^{\mathrm{b}}$, Mark \\ Cresitello-Dittmar ${ }^{\mathrm{a}}$, Stephen M. Doe ${ }^{\mathrm{a}, 2}$, Rick Ebert ${ }^{\mathrm{c}}$, Janet D. Evans ${ }^{\mathrm{a}}$, Patrick Norris ${ }^{\mathrm{d}}$, Olga Pevunova ${ }^{\mathrm{c}}$, Brian \\ Refsdal $^{\mathrm{a}}$, Brian Thomas ${ }^{\mathrm{d}}$, Randy Thompson $^{\mathrm{b}}$ \\ ${ }^{a}$ Smithsonian Astrophysical Observatory, 60 Garden St. Cambridge, MA 02138 \\ ${ }^{b}$ Space Telescope Science Institute, 3700 San Martin Dr. Baltimore, MD 21218 \\ ${ }^{c}$ Infrared Processing and Analysis Center, 770 South Wilson Ave. Pasadena, CA 91125 \\ ${ }^{d}$ National Optical Astronomy Observatory, $950 \mathrm{~N}$ Cherry Ave. Tucson, AZ 85719
}

\begin{abstract}
Iris is an extensible application that provides astronomers with a user-friendly interface capable of ingesting broad-band data from many different sources in order to build, explore, and model spectral energy distributions (SEDs). Iris takes advantage of the standards defined by the International Virtual Observatory Alliance, but hides the technicalities of such standards by implementing different layers of abstraction on top of them. Such intermediate layers provide hooks that users and developers can exploit in order to extend the capabilities provided by Iris. For instance, custom Python models can be combined in arbitrary ways with the Iris built-in models or with other custom functions. As such, Iris offers a platform for the development and integration of SED data, services, and applications, either from the user's system or from the web. In this paper we describe the built-in features provided by Iris for building and analyzing SEDs. We also explore in some detail the Iris framework and software development kit, showing how astronomers and software developers can plug their code into an integrated SED analysis environment.
\end{abstract}

Keywords: data abstraction, method: data analysis, object-oriented programming, software frameworks, spectral energy distribution, virtual observatory tools

\section{Introduction}

The emission processes of astronomical objects (e.g., stars, galaxies, quasars) are reflected in the spectral energy distribution (SED) of the radiation detected by astronomers with a variety of telescopes and instruments. Astronomers use this information to infer the physical properties of the source by comparing the detected SED with different emission models. Methods for these studies have been developed by several communities in astronomy, focused on either a particular type of source, or quite often on a particular region of the emission spectrum (e.g., radio, optical-IR, X-ray). These focused tools typically require different input formats and imply the use of wavelength specific units, as well as being optimized for particular models to compare the SED with. However, the most complete picture of any emission phenomena requires the use of the most complete information base. Modern widefield ground and space telescopes, and the availability of

\footnotetext{
* Corresponding author

Email address: olaurinodcfa.harvard.edu (Omar Laurino )

${ }^{1}$ Present affiliation: McGill University, 3600 University St. Montréal QC, Canada H3A 2T8

${ }^{2}$ Present address: 4 Lafayette Drive, East Walpole, MA 02032
}

data from multi-wavelenth archives, allow in principle to build and study broadband SEDs for any kind of astronomical object. However, a tool that can efficiently and powerfully make use of this information requires a nonwavelength-specific approach.

The International Virtual Observatory Alliance (IVOA; Quinn et al., 2004) provides a set of standards and protocols that facilitate interoperability among astronomy-related services and tools. These IVOA specifications can be implemented to enable generalized SED analysis, regardless of the spectral regime and objects being studied.

In order to design effective applications, one wants to leverage IVOA standards without exposing the complexity and technicality of their specifications to the users. Also, while application developers implement many desired features, it is useful, and sometimes required, to provide hooks for users and third party developers to extend the application's functionality without requiring knowledge of standards themselves. Designing such an application, like a general SED analysis tool, thus becomes an exercise in designing a framework that implements some basic, effective functionality for a wide set of use cases, while being highly extensible. 
Iris, the Virtual Astronomical Observatory (VAO; Berriman et al. (2012) SED analysis tool, is such an IVOAenabled desktop application. With Iris, users may populate SEDs with data from files, built-in portals to data archives, and other Virtual Observatory (VO) applications. Users can interactively visualize and edit SEDs, and fit SEDs with fine-tuned modeling features. Iris provides a suite of astrophysical models, but also lets users import custom models and template libraries. All front-end features of Iris completely hide the underlying technical IVOA specifications from the user.

While implementing IVOA standards and protocols, we took advantage of existing astronomy software, namely Specview (Busko, 2002) for the visualization and fitting user interfaces, the NASA/IPAC Extragalactic Database (NED) SED Service ${ }^{3}$ for data acquisition, and Sherpa (Freeman et al. 2001, Refsdal et al., 2009) for the modeling and fitting engine. Along with these components, new ones, like the SED Builder, were developed specifically for Iris (Doe et al. 2012, Laurino et al., 2013).

Iris was developed inside the framework of the VAO science applications: the different components were contributed by developers from the Smithsonian Astrophysical Observatory, the Space Telescope Science Institute (STScI), and the NASA Infrared Processing and Analysis Center (IPAC). Quality assurance and testing were led by team members at the National Optical Astronomy Observatory and STScI.

In this paper we present the Iris application, design, and extensible architecture. In Section 2 we briefly explore the landscape of SED applications and analysis tools that Iris joined, and provide an example use-case of Iris. We explore how astronomers can include their own models or templates as Python functions in Section 3. An introduction to Iris' general architecture (the Iris stack) is illustrated in Section 4. A more detailed overview of the Iris extensible framework design (Section 5) is followed by a detailed description of the more advanced Iris capabilities (Section 6). Finally, we describe the Iris software development kit, including a "How-to" on extending Iris with plug-ins (Section 7). Sections 5 and 7 are targeted to software developers.

The paper refers to version 2.0.1 of Iris. Iris can be downloaded as a binary archive for OS X and Linux 4 and the source code is hosted on GitHub as a public repository 5 .

\section{SED Analysis with Iris}

Fitting spectral energy distributions enables astronomers to estimate fundamental properties of various astronomical objects. In galaxy evolution studies, for example,

$\sqrt[3]{\text { http://vo.ned.ipac.caltech.edu/SED_Service/ }}$

4 http://cxc.cfa.harvard.edu/iris/latest/download/

5 https://github.com/ChandracXC/iris stellar mass, star formation rates, dust content, and redshift are often derived from galaxy SEDs (e.g. Sawicki and Yee (1998), Shapley et al. (2001), Robitaille et al. (2007), and many others). Accretion disks surrounding supermassive black holes, x-ray binary and young stellar objects can be studied by fitting models to the host objects' SEDs, extracting information like accretion rates, disk geometry, and disk temperature (e.g., Czerny and Elvis, 1987, Vrtilek et al., 1990, Chiang and Goldreich, 1997, Robitaille et al. 2006). Stellar SED analysis can recognize mid IR excess, which may indicate circumstellar disks (Lagrange et al. 2000, Chen et al., 2005). As these examples show, SEDs are widely used throughout astronomy.

With ever increasing wide-field surveys and datasets over the years, astronomers have been able to use multiwavelength SEDs more frequently for their research. As such, many robust SED analysis codes have been created to help astronomers model, fit, and derive physical quantities from SEDs (Walcher et al. 2011, Conroy, 2013). These widely-used codes implement a diverse set of methods, for instance: inversion (e.g., STARLIGHT Cid Fernandes et al., 2004 and PAHFIT Smith et al., 2007]), principal component analysis (e.g., Budavári et al. 2009), $\chi^{2}$-minimization codes (e.g., Le PHARE Arnouts et al. 1999, Ilbert et al. 2006 and HyperZ Bolzonella et al. 2000]), and Bayesian inference (e.g., BPZ Benítez, 2000], VOSA Bayo et al. 2008, and GalMC Acquaviva et al. 2011]).

Most widely used fitting packages are tailored for specific data sets or spectral ranges (such as PAHFIT and STARLIGHT), providing robust fitting methods and results. They require the data to be in a specific format with specific units in order for the tool to work properly. When fitting a broadband SED that spans over decades in the spectrum, the astronomer will typically gather datasets from different public archives and colleagues in order to add such data to their own. More often than not, the datasets are stored in different file formats and units. The user must provide their own methods to extract the necessary data from each file, homogenize the units, and output a file in the format supported by the tool; converting the data to a supported format may easily become a tedious task with each additional dataset.

While SED analysis tools often have different input formats from each other, they effectively require the same information to run. Whether datasets are stored in a FITS file, a tab-separated ASCII table, or a VOTable (Ochsenbein et al. 2011) coming from a VO data discovery application, they are all serializations of the same, global, abstract, scientific model of photometric measurements for astronomical sources.

By employing a standardized definition of such models, Iris streamlines the process of building SEDs for analysis. In other terms, one of the goals of Iris is to make SED building a painless and straightforward process, letting the scientist focus on the sophisticated and original parts of the scientific work-flow: data analysis, hypothesis testing, 
Table 1: Supported file formats. Native formats are automatically loaded into Iris. Supported formats require some user input to map the file data to the spectral and flux information.

\begin{tabular}{|c|c|c|}
\hline \multirow{3}{*}{ 离 } & Format & Description \\
\hline & VOTable & XML-based format, text or binary following IVOA Spectrum Data Model v1.0, 1.1, or 1.2. \\
\hline & FITS & $\begin{array}{l}\text { Series of HDUs }{ }^{\mathrm{a}} \text { with text header and text or binary data extensions following IVOA Spec- } \\
\text { trum Data Model v1.0,1.1, or } 1.2 \text {. }\end{array}$ \\
\hline \multirow{6}{*}{ 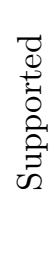 } & VOTable & XML-format, text or binary. \\
\hline & FITS & Series of HDUs ${ }^{\mathrm{a}}$ with text header and text or binary data extensions. \\
\hline & ASCII Table & Text file with columns separated by spaces and/or tabs. \\
\hline & CSV & Text file with columns separated by commas (first row may contain column names). \\
\hline & IPAC & A custom bar-separated text format by IPAC. \\
\hline & TST & Tab Separated Table (comments are ignored, metadata is in key, value pairs). \\
\hline
\end{tabular}

a Header Data Units.

Table 2: Supported SED units. Iris can read, write, and/or plot data in the spectral and flux units listed in this table. Italicized units are only available for plotting.

\begin{tabular}{|c|c|}
\hline Spectral Axis & Flux Axis \\
\hline$\AA$ & $\mathrm{erg} / \mathrm{s} / \mathrm{cm}^{2} / \AA$ \\
\hline $\mathrm{nm}$ & $\mathrm{erg} / \mathrm{s} / \mathrm{cm}^{2} / \mathrm{Hz}$ \\
\hline$\mu \mathrm{m}$ & photon $/ \mathrm{s} / \mathrm{cm}^{2} / \AA$ \\
\hline$m m$ & photon $/ \mathrm{s} / \mathrm{cm}^{2} / \mathrm{Hz}$ \\
\hline $\mathrm{cm}$ & Watt $/ \mathrm{m}^{2} / \mu \mathrm{m}$ \\
\hline $\mathrm{m}$ & $W a t t / \mathrm{cm}^{2} / \mu m$ \\
\hline $\mathrm{eV}$ & Watt $/ m^{2} / n m$ \\
\hline $\mathrm{keV}$ & $\mathrm{Watt} / \mathrm{m}^{2} / \mathrm{Hz}$ \\
\hline $\mathrm{MeV}$ & Rayleigh/A \\
\hline $\mathrm{Hz}$ & $\mathrm{Jy}$ \\
\hline $\mathrm{kHz}$ & mJy \\
\hline $\mathrm{MHz}$ & $\mu J y$ \\
\hline \multicolumn{2}{|l|}{$\mathrm{GHz}$} \\
\hline $\mathrm{THz}$ & $\mathrm{AB}$ mag \\
\hline $1 / \mu m$ & $\mathrm{ST} \mathrm{mag}^{\mathrm{a}}$ \\
\hline \multirow{3}{*}{$\begin{array}{l}\mathrm{km} / \mathrm{s} @ 21 \mathrm{~cm} \\
\mathrm{~km} / \mathrm{s} @ 12 \mathrm{CO}\end{array}$} & \\
\hline & Jy Hz \\
\hline & $\mathrm{erg} / \mathrm{s} / \mathrm{cm}^{2}$ \\
\hline $\begin{array}{l}\mathrm{ST}=-2.5 \log _{10} \\
\text { the source flux } \\
\text { wavelength. }\end{array}$ & $\begin{array}{l}f_{\lambda}-21.10, \text { where } f_{\lambda} \text { is } \\
\text { nsity expressed per uni }\end{array}$ \\
\hline
\end{tabular}

and knowledge extraction.

Following VO efforts to combine data services and applications seamlessly, Iris offers an interface for building large broadband SEDs from different sources in various data formats, while providing robust fitting methods and interactive visualization capabilities using existing astronomical software. It is important to stress that this is not only a matter of ingesting non-standard files, but also to allow scientists to create standardized versions of their datasets: the improved interoperability enables more tools, inside or outside Iris, to load and interpret such datasets with minimal user intervention.

Much effort has been put into making Iris lenient on data format. While natively supporting VO-compliant files (properly annotated VOTable and FITS files), Iris can ingest ASCII, CSV, and other table-like formats as well with some extra user input. Table 1 describes the file formats that can be read into Iris. Users may also seamlessly transfer data from other VO applications or data archive services through SAMP, the Simple Application Messaging Protocol (Taylor et al., 2011). Moreover, Iris can read, write, and display SED data in a variety of commonly-used units, which are listed in Table 2, with minimal user effort.

But more importantly, Iris provides standardized views of the integrated datasets to its clients, whether they are built-in components, third party plug-ins, or external applications.

\subsection{A Use Case}

In this section, we present a brief, illustrative use-case of Iris to showcase its main features. We outline the analysis of the broadband SED of flat spectrum radio quasar (FSRQ) object PKS 1127-14 (see Błażejowski et al. 2004), and save the results to file.

For details on the Iris features introduced in this usecase, see Section 6.

An Iris session begins with populating a SED by clicking on the SED Builder icon on the Iris desktop. A user loads a local ASCII file of PLANCK data, a WISE dataset from TOPCAT (Taylor, 2005, ascl:1101.010 through a SAMP message, and all data associated with PKS 112714 in NED with the NED SED Service portal. The user also uses the built-in Italian Space Agency Science Data Center ${ }^{7}$ (ASDC) query tool to find optical/UV data for PKS 1127-14, and adds it to the SED (see Figure 1).

Data are converted to a single set of units on the fly, and displayed in the SED Viewer. The user can switch the spectral and flux axes between a variety of commonlyused SED units, e.g., one can switch from Jy vs. $\mu \mathrm{m}$ to

\footnotetext{
${ }^{6}$ ascl: Astronomy Source Code Library

7 http://www.asdc.asi.it/
} 


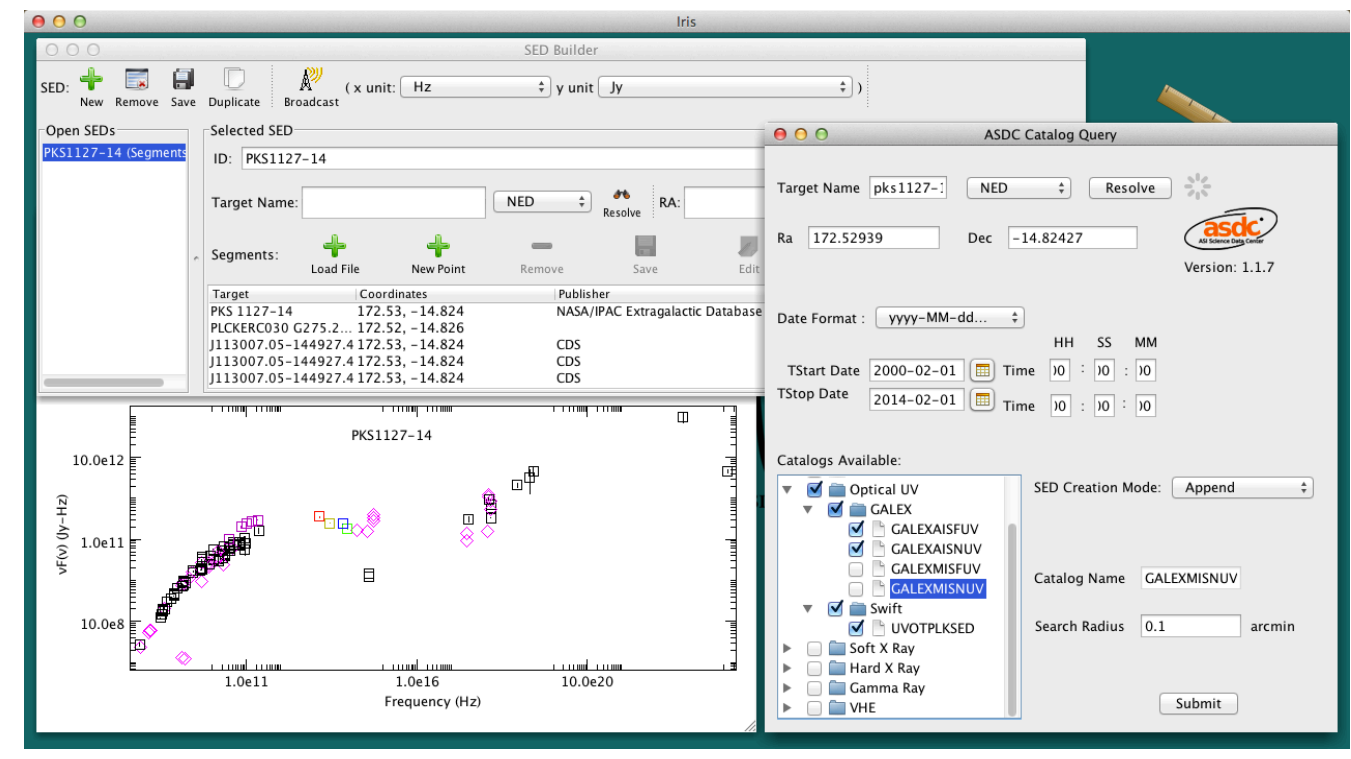

Figure 1: Building the SED of blazar PKS 1127-14 in Iris. Top-left: Data from the NED SED Service, a local file, and from TOPCAT are managed in the SED Builder. Bottom-left: The various data segments plotted in $\nu \mathrm{F}(\nu)$ units inside the SED Viewer. Squares show data with flux uncertainties, whereas the pink diamonds denote points without associated uncertainties. Each segment in the SED Builder is plotted in a different color. Black squares are data taken from NED; the pink squares in the radio are the data from PLANCK; and the red, yellow, blue, and green squares in the near-IR are the four WISE bands. Right: An ASDC Data Query form for PKS 1127-14. The user searches for data between specified dates and available instruments (Swift and GALEX in this case). The data have been added to the open SED PKS 1127-14

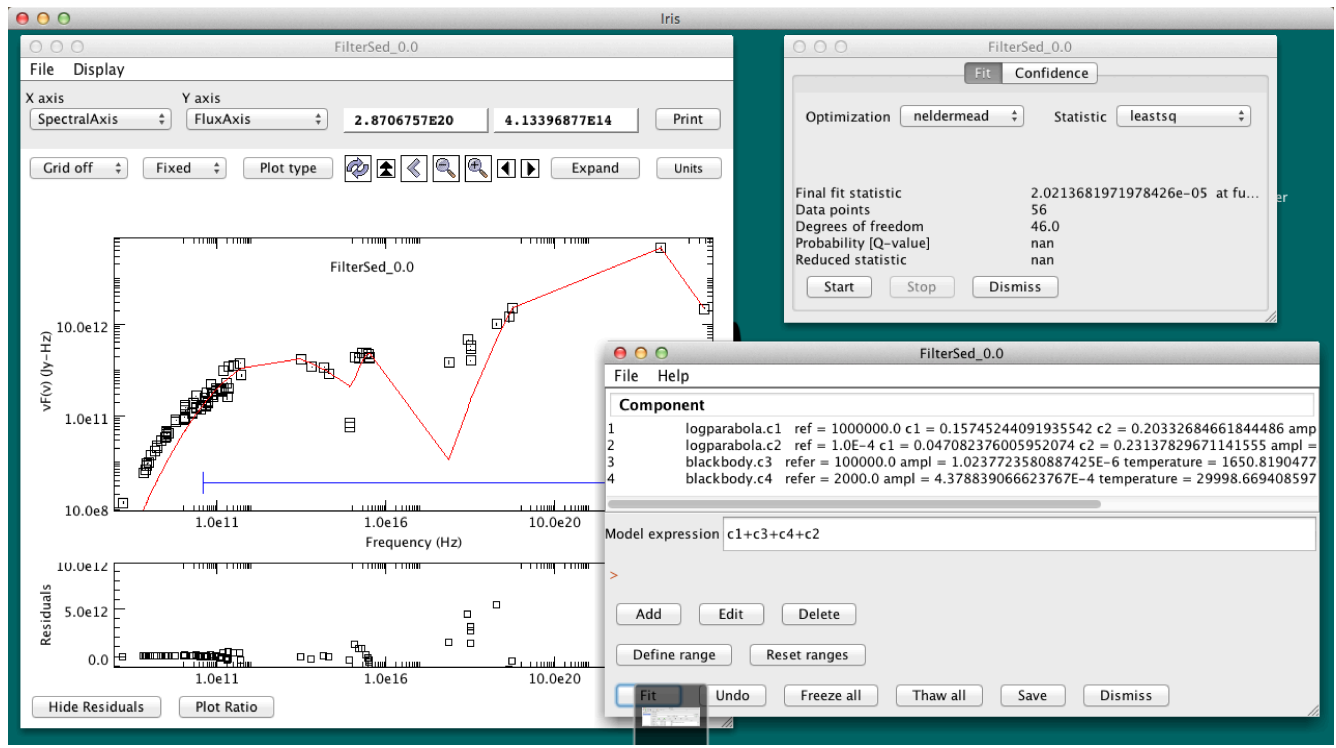

Figure 2: Fitting Visualization. Visualization of a linear combination of log-parabolas and blackbody distributions for FRSQ blazar PKS 1127-145, fit with Nelder-Mead optimization and least-square statistics. Left: The best fit linear combination overlaid on the SED data as a red curve. The blue line shows the spectral range over which the data were fit. Below the main plot are residuals of the fitted curve, in dex units. Top-right: The fitting options and results. Here, the user chooses between Nelder-Mead, Levenberg-Marquardt, and Monte-Carlo (Differential Evolution, Storn and Price 1997) optimization and various least square and $\chi^{2}$ statistics. The fit statistics are reported here after the fit has been performed. Bottom-right: The Fitting Tool window. The model components used in the fit and their fitted parameter values are listed in the Components field. Below that is the Model Expression, in which the components are linearly combined. Note that components are referenced by the $c \#$ suffix of the component name. 
JyHz vs. Hz. The Metadata Browser - an interactive table of the SED data - allows the user to interactively inspect and filter out data points by hand or with Boolean expressions.

The user also employs the Science Tools, an Iris built-in component that lets the user cosmologically redshift SEDs, interpolate SED data, and calculate integrated fluxes of SEDs through photometric filters or user-defined passbands. In particular, the user shifts PKS 1127-14 from its observed redshift at $z=1.18$ to rest frame using the Redshift tool before fitting the SED.

The user then filters out all the points devoid of errors using the metadata browser filtering features.

When the user is done building and editing the SED, the user begins the fitting session. With the fitting tool, the user can build a model expression as an arbitrary combination of model components. Choosing from a list of built-in astrophysical and mathematical models, the user fits PKS 1127-14 with a linear combination of four models: two logarithmic parabolas to model the radio synchrotron and inverse Compton radiation (Massaro et al. 2006, Tramacere et al., 2009), and two blackbodies to approximate the models for the hot dust component and accretion disk of the blazar (Dermer and Schlickeiser, 2002). The fit is performed using Nelder-Mead optimization and least square statistics. The user has fine control over the parameters, including setting initial values, the range of the values, freezing and thawing parameters, and linking model parameters to other parameters in the model expression; the user also controls the spectral ranges over which to fit the models. Finally, confidence intervals are computed for the overall model parameters.

Figure 2 shows the final model for PKS 1127-14 overlaid on the input data and, in the lower panel, the fit residuals.

When the user is satisfied with the fitting results, the user saves an XML-style file of the model that can be reread into Iris and fit to other SED data. The user also saves the fit results to a text file, that shows the parameters of the fit and the details about each model component, with the best-fit parameter values.

\section{User Models and Templates}

Keeping with our requirements of developing an extensible SED analysis tool, we provide a user interface for adding custom models, templates, and template libraries for the fitting engine to use in a Custom Fit Models Manager.

Sherpa, Iris' fitting engine, provides command line functions for users to add their own models and templates to a Sherpa session. We wrap a graphical user interface (GUI) around such functions for streamlined integration and user-friendliness. The user provides the full path to the directory where the models and templates exist, as well as information about the parameters. Installing a model saves a copy of the model files in the user's home directory (in / . vao/iris/components), allowing the user to apply the models in future sessions.

\subsection{Custom Python Functions}

Iris accepts custom models as Python functions stored on the user's disk. Any number of functions can be stored in a single file. The function implementing the model must take two parameters: the first is an iterable of the model parameters, the second is a placeholder for the spectral axis, $x$, in units of Angstroms. For example, a model file for a modified black body $B_{\nu}(T)\left(\nu / \nu_{0}\right)^{\beta}$ could be defined as in Listing 1 .

User models can be arbitrarily combined with other custom or preset model functions when using the Iris fitting tool.

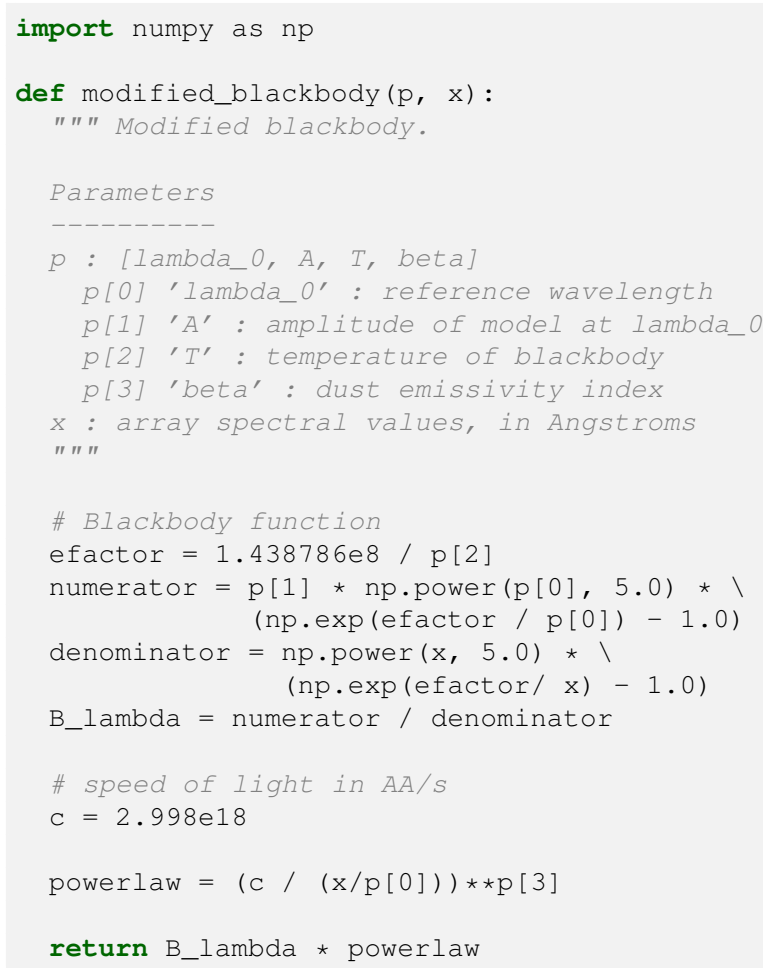

Listing 1: Example of a user-defined model that can be dynamically loaded into Iris. The code, written as a Python function, implements a modified blackbody and can be combined in Iris with other built-in and custom components. Backslashes indicate line continuations.

\subsection{Table Models}

A table model is a single template, having just the $x$ and $y$ coordinates. Iris accepts two column ASCII files as table models, following the convention where the first column is the spectral values and the second contains the fluxes. The spectral and flux units must be in Angstroms and photons $/ \mathrm{s} / \mathrm{cm}^{2} / \AA$, respectively ${ }^{8}$ The fit returns the

8 While Iris ingests many other units (see Table 2, the Custom Fit Model Manager is independent of Iris's units handler and only accepts files with spectral values in Angstroms and the flux in photons $/ \mathrm{s} / \mathrm{cm}^{2} / \AA$ 
normalization constant (or amplitude) of the model.

\subsection{Template Libraries}

The template model is essentially a list of table models with parameters other than the amplitude. Like the load_template_model function in Sherpa, the user must create an index file that lists the parameter values of the templates and the full path to the template those parameter values describe (see Listing 2 for an example). Sherpa uses a grid-search method to find the best-fit template. The parameters grid is created using the values provided in the index file.

$\begin{array}{lll}\text { \# INDEX } & \text { REFER } & \text { MODELFLAG FILENAME } \\ 0.0 & 5000 & 1 / \text { data/sed_temp_index-0.00.dat } \\ -0.10 & 5000 & 1 / \text { data/sed_temp_index-0.10.dat } \\ -0.25 & 5000 & 1 / \text { data/sed_temp_index-0.25.dat } \\ -0.35 & 5000 & 1 / \text { data/sed_temp_index-0.35.dat } \\ -0.50 & 5000 & 1 / \text { data/sed_temp_index-0.50.dat }\end{array}$

Listing 2: Example of template library definition file. Template library definition files are in ASCII format.

\section{The Iris Stack}

The Iris stack (Figure 3) shows how one can put the technical IVOA specifications to work for scientists through higher and higher layers of abstraction: the details of the Virtual Observatory standards and protocols lie in the lowest layer, the internals of the Iris building blocks lie in the middle layer, while the top layer expresses high-level user-oriented features.

A reader without any knowledge of programming, let alone of the VO specifications, should understand the labels used in the top layer of the diagram and their components (e.g., Fitting Tool and Redshifting), as long as they have some knowledge of astronomical SEDs. On the other hand, a developer would find words like framework, service, and manager quite familiar, while it takes a VOsavvy person to decode the acronyms at the bottom of the diagram ${ }^{9}$

This architecture enables different entry points for the different audiences of the application. Core developers work at all levels of the stack, but need to lay out the foundations on top of the standard specifications; third party developers use the middle-level abstractions offered by the Iris framework, while end users can limit their interaction to familiar astronomical concepts through the

${ }^{9}$ SAMP, the Simple Application Messaging protocol was already introduced, DM stands for Data Model and the SpectrumDM is introduced later in the paper. UTYPEs are labels used in some file formats (like VOTable, introduced later) to tag data elements according to a Data Model. SSAP stands for Simple Spectral Access Protocol and is implemented by services that provide access to spectral datasets, including SEDs, and is also introduced later in the document.

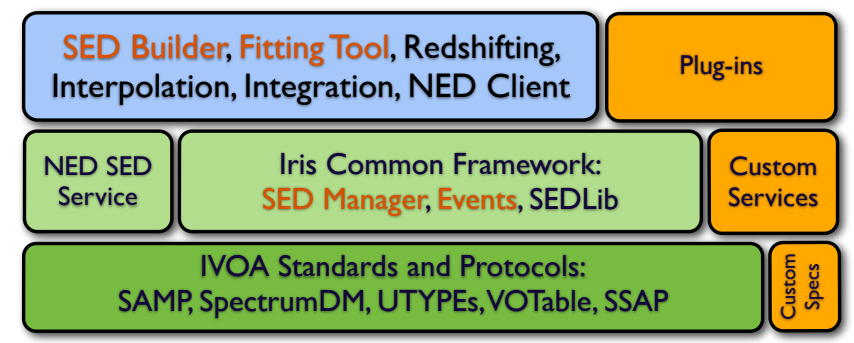

Figure 3: The Iris Stack. With its architecture Iris allows developers to create components using higher and higher abstractions on top of web services, desktop applications, and Virtual Observatory standards and protocols. The technical specifications lie on the bottom, a middle layer provides abstractions useful to developers, and the user is only exposed to the science features represented by the top layer. Users can also plug their code in as Python functions. The result is aimed to be both user- and developer- friendly. Notice that custom services can be built on top of custom specifications, but also on top of IVOA standards. Similarly, plug-ins can use custom services, but they also probably use the Iris Common Framework. The top layer components (built-in Iris features and plug-ins) provide the user with scientific features within Iris.

application's user interface. End users can also plug in their modeling code and upload templates libraries to Iris.

The color code in Figure 3 adds a different dimension to this diagram and taps into a different characteristic of the Iris architecture: extensibility. In particular, scarlet letters denote extensible components of the architecture, i.e., components that offer hooks into the Iris architecture to users and developers. The orange boxes, on the other hand, express components that were not part of the Iris design, but that can be used in Iris as plug-ins, possibly providing interfaces to access non-standard services. Some of these plug-ins, along with a description of the design of the Iris Software Development Kit, will be introduced in Section 7

The dark green box denotes IVOA sanctioned standards. Blue denotes components that are built-in in Iris and light green boxes denote components that were developed in or for Iris 10

This architecture was also driven by a more abstract requirement: our team was distributed, with developers and managers working from different institutions with different tools and practices (Evans et al. 2012). Moreover, wanting to reuse existing software instead of reinventing the proverbial wheel, we had to integrate different existing software components in a seamless way. So, the Iris stack provided not only a clean and robust architecture for users and third party developers, but was also useful in enabling a distributed team of part-time developers to

\footnotetext{
${ }^{10}$ While the NED SED service was developed independently of Iris, its IVOA-compliant interface was part of the Iris project, along with the development of a dedicated client in Iris itself.
} 
work in parallel, reducing the overall project risk.

In summary, the Iris framework was designed to address several different requirements: (i) functional requirements gathered by the Iris team's lead scientists; (ii) functional requirements unknown at development-time; (iii) the distributed nature of the Iris development team; and (iv) interoperability between several existing tools and services.

The Iris stack offers a non-technical view of the Iris architecture and design. While the stack shows effectively how we tried to abstract end users and developers from the VO specifications and from the specifics of the Iris internals, the stack does not express the technical solutions that we employed to achieve such extensible architecture and to meet the aforementioned requirements. More detail is provided in some of the following sections.

\section{The Iris Architecture}

In order to minimize the risk derived from the requirements listed in the previous section, we backed Iris with a loosely coupled architecture through a design pattern called Inversion of Control (Johnson and Foote, 1988).

But it was not just a matter of risk management. Inversion of Control supports the implementation of liquid requirements, i.e., a finite set of predetermined requirements plus an indefinite set of custom requirements to be implemented by users, at least in some simple cases or, for more advanced features, by third party developers.

The architecture that supports the implementation of such requirements has different components that can be mapped to the Model-View-Controller (MVC) design pattern.

SEDLib This basic I/O library provides classes for the Model components of MVC. Unsurprisingly, SEDLib does so by implementing a Data Model specification defined by the IVOA. The Data Model defines both the logical breakdown of spectral datasets, and the serialization in some standard file formats. So, on the one hand, SEDLib can perform the basic read/write operations on spectrophotometric files, while on the other the library provides the data structures that client components can use and exchange.

SEDManager The MVC Controller role is played in Iris by the SEDManager, which itself is defined as an Interface. The manager works as a data storage for SEDLib instances that the different Iris components can share.

Components The actual Iris functionality is implemented by the Iris Components. They can be seen as the Views in the MVC pattern (or, more generally, they can provide any number of Views), since they present the data stored in the Controller to the user, query the Controller itself, and act upon the Models, i.e., the SED objects provided by SEDLib.
Events Views can be notified of changes in the Models by Events, if they implement the relative Listener interface and have been registered to the Events Queue. Events usually have a payload with more information about their content, and a pointer to the Model instances involved.

In summary, Components (Views) can be completely disentangled from each other and interact indirectly through the sole common interface represented by the SEDManager (Controller), which in turn stores the SED objects (Model). Dynamic changes in the system are notified to all interested agents (Listeners) via specific Events.

Components are thus agents that cooperate by attaching themselves to a common bus where the SEDManager provides the memory, and Events guarantee the flow of information (see Figure 4).

\subsection{Inversion of Control}

We achieve loose coupling by an extensive use of Java Interfaces: components, events, and event listeners, for example, are all defined by interfaces whose implementation can, to some extent, be freely interchangeable.

Moreover, Inversion of Control is employed to decouple the implementation of components from the run time context (see Figure 5). Methods in the Interface are callbacks, and some of these callbacks get Interface-typed arguments that provide them context instances during application execution. For this reason, this pattern is also sometimes referred to as Dependency Injection ${ }^{11}$

Consider, for example, Iris Components: they are the main providers of Iris functionality, and they can correspond to buttons and menu items on the Iris desktop, loggers, data handlers, etc. They must implement the IrisComponent interface, listed in Listing 3 .

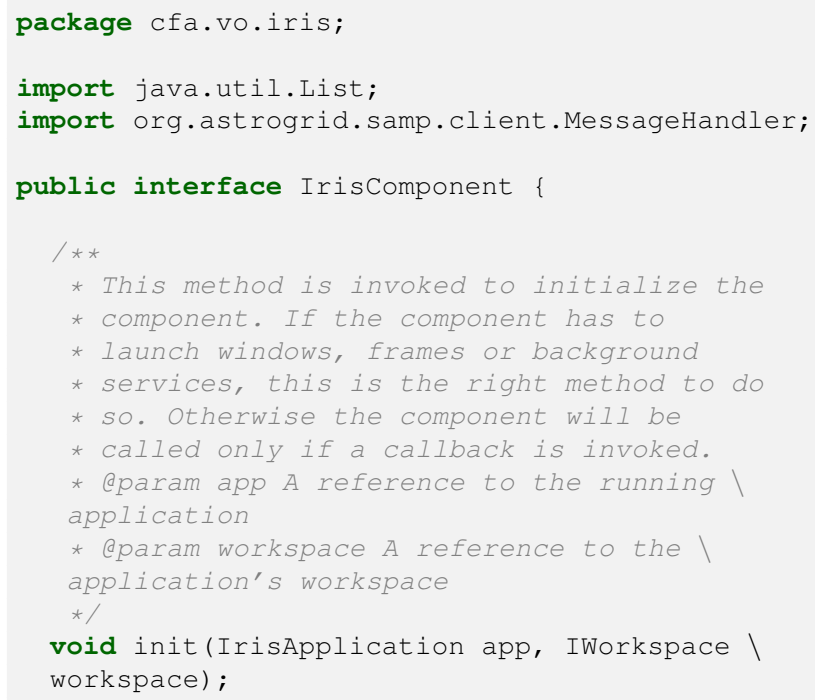

${ }^{11}$ There is, to be precise, a subtle but significant difference between Dependency Injection and Inversion of Control, the first effectively being a special case of the second. 


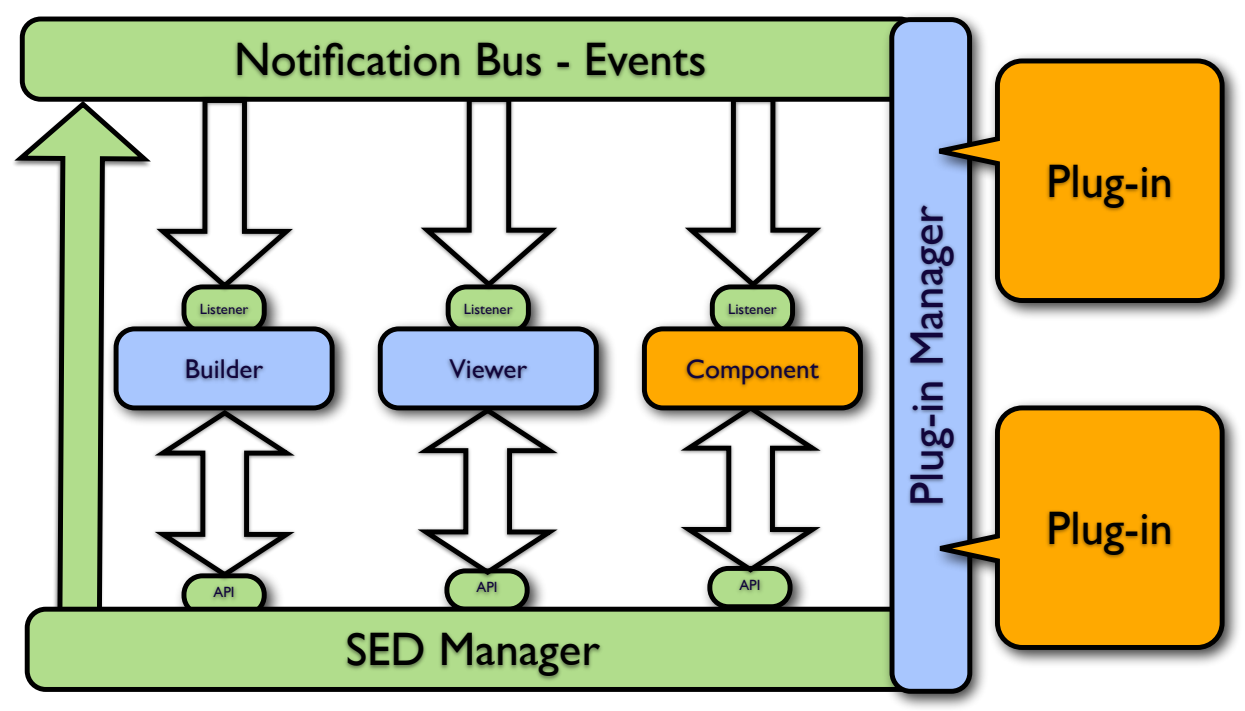

Figure 4: The Iris loosely coupled, extensible architecture. Information freely flows among built-in and third-party components provided as plug-ins. A SED Manager gives components access to the state of the SEDs in the user's workspace, while dynamic changes in such state are announced through events that are notified to the subscribed listeners. A plug-in manager allows users to install and uninstall plug-ins on the fly.

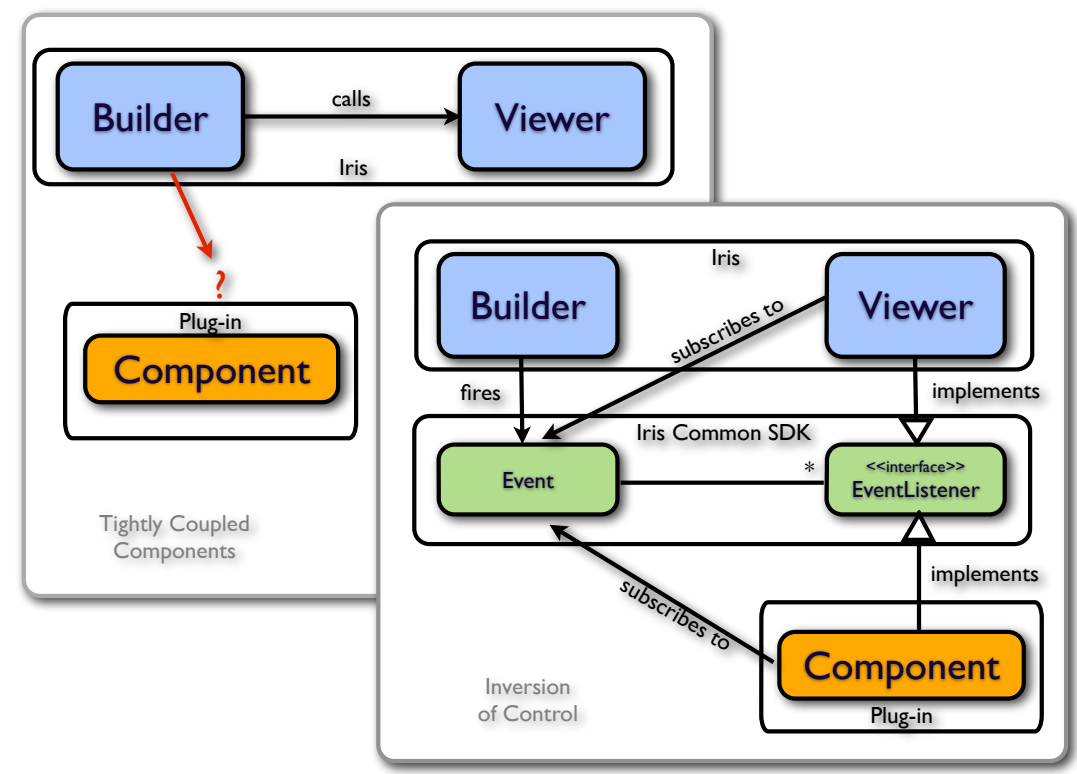

Figure 5: Inversion of Control. IoC is a design pattern that promotes decoupling of software components so that they can easily be replaced by different implementations, with the actual binding often happening at run-time according to some configuration. This pattern, however, also allows new components to be added at any time during the application lifecycle: a common framework (Iris Common) can be shared as a middle layer among implementations, and a container (the Iris application) can bind components together on the fly. Components can subscribe to events and react to them. 


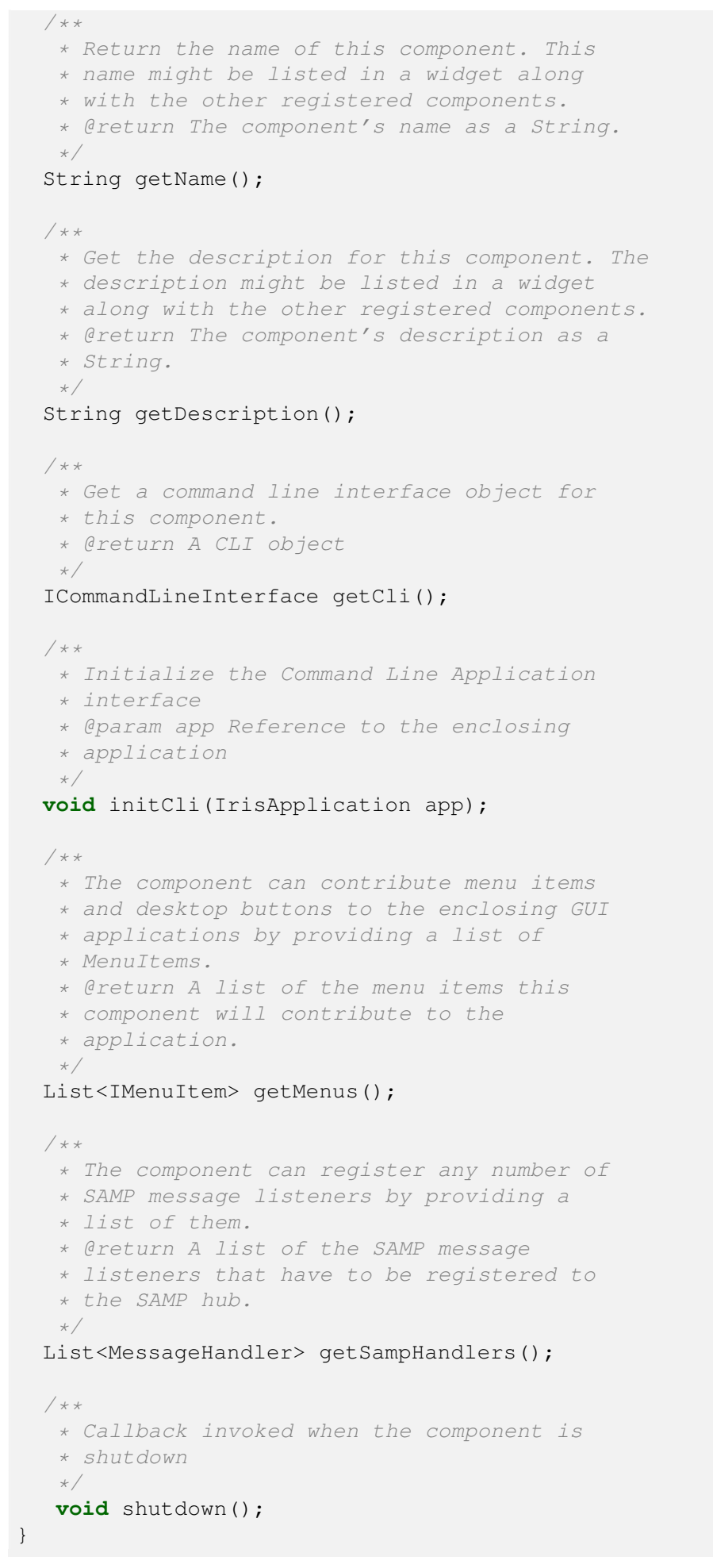

Listing 3: This snippet of Java code represents the main interface that all components in Iris have to implement, and how dependencies get injected into the components at run-time. Backslashes indicate line continuations.

At startup the Iris application reads the list of Components to be initiated, and calls their init call-back, which in turn is passed useful information like a reference to the SEDManager, or hooks to the application environment.
The advantages of this architecture are both functional and non functional. The architecture helped our heterogeneous development team to work in a loosely coupled way, reducing the overall project risk, and also provided the extensible framework we were seeking in the first place. As a matter of fact, plug-ins that can be loaded at run time implement the same interfaces that the built-in components do, and they are instantiated in exactly the same way. The only difference is in the timing: built-in Components get instantiated when the application itself is initialized, while plug-ins can be instantiated and discarded at any time during the application execution.

\section{Iris Built-in Components}

In the previous section, we discussed the architecture of Iris and how the different Components in Iris communicate. Each Component performs one or more SED-related tasks in Iris, like building SEDs from multiple sources and fine-tuned SED modeling. Here, we discuss what the Components do in terms of the science domain, including descriptions of the autonomous software used to build Iris: Specview, Sherpa, and the NED SED Service.

\subsection{SED Builder}

Users manage SEDs through the SED Builder (Figure 6). From the Builder, users can add, edit, remove, and save SEDs. Users can also transfer data seamlessly to other VO-enabled applications through SAMP messages from the Builder. Any number of SEDs can be analyzed in an Iris session. Each SED has a unique identifier that is set by default when a new SED is created, but can be changed by the user. The user switches between SEDs by clicking on a SED name in the Open SEDs field; the visualizer will automatically update to the selected SED.

SEDs are built and managed in Segments, which are groups of (spectral, flux) coordinates. For example, a spectrum is considered a Segment; the results of a NED SED Service query are also handled as a Segment. In general, anything from a single photometric point to an entire SED can be considered a Segment, with all the points sharing some if not all of the metadata.

Clicking on a SED in the Open SEDs field will show all the Segments that populate that particular SED. SED Builder shows where the Segment data came from, the recorded RA and Dec of the Segment, and the number of points in the Segment. Segments can be handled separately from other Segments in the SED; users can add, edit, remove, and save a subset of Segments selected from a SED.

\subsubsection{Importing data}

As described in Section 2, Iris accepts data from a variety of sources, and is lenient on the data format. Figure 7 illustrates that Iris imports data from built-in data archive 


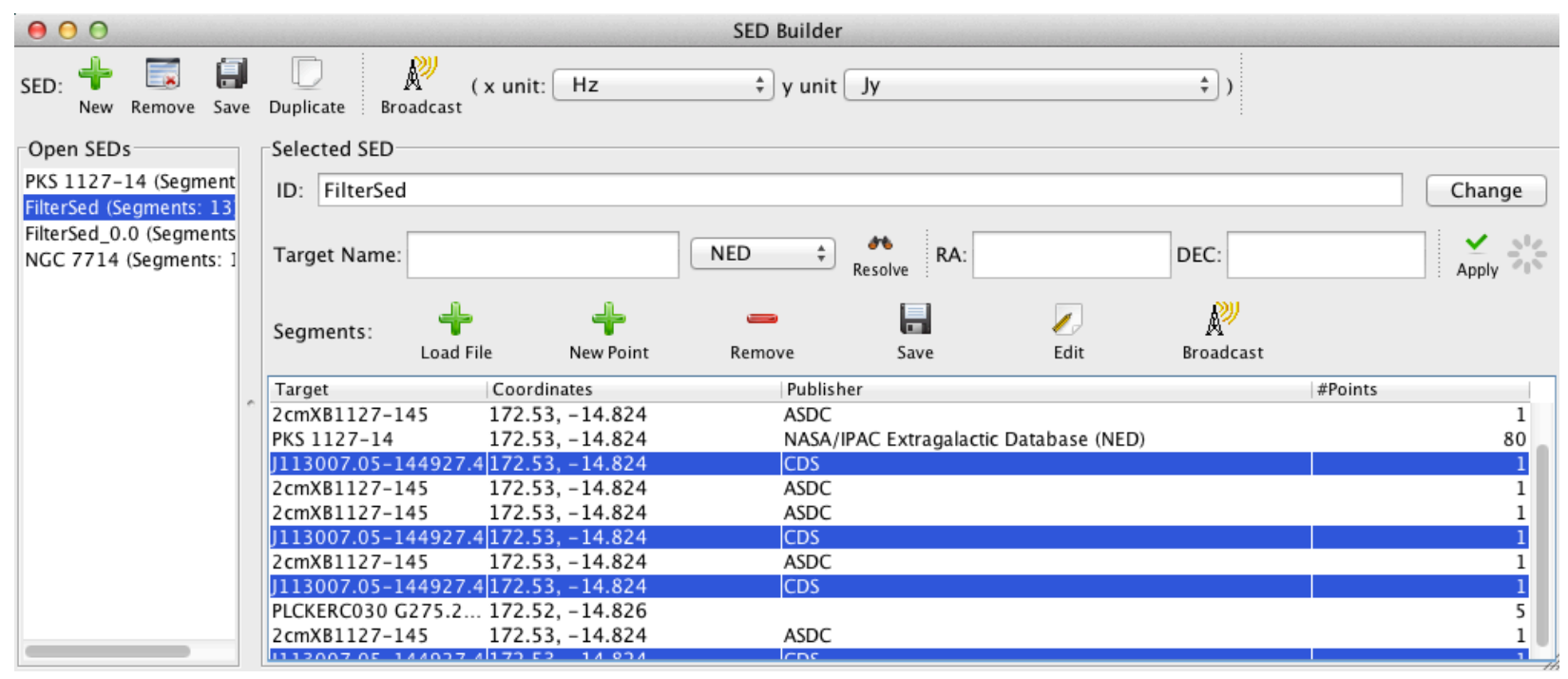

Figure 6: SED Builder. An example view of the SED Builder. On the left side of the window is a list of SEDs open for analysis; in this case, FilterSed is selected. The Segments or components that constitute FilterSed are shown in the Segments section. Segments may be managed separately. Highlighted SEDs or Segments may be edited, removed, saved, or broadcast to an external SAMP-enabled application. New Segments and SEDs are added to the Iris session through the SED Builder.

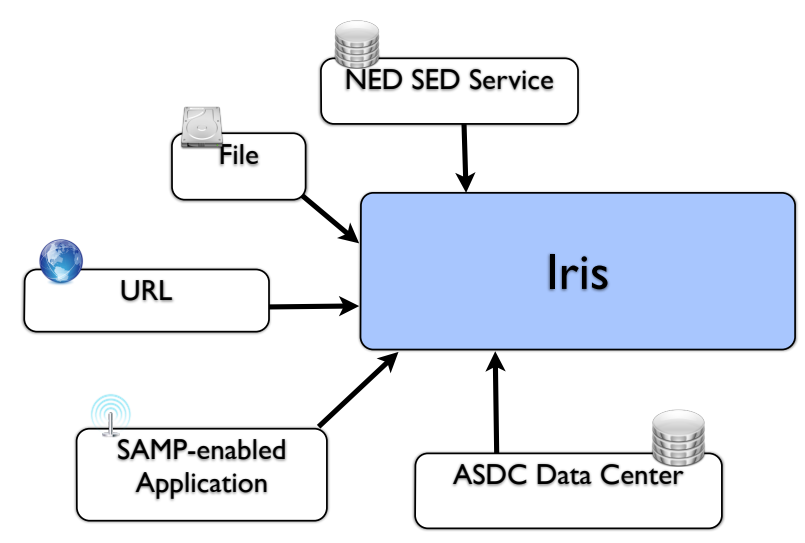

Figure 7: Available Data Sources. Users may import data from the built-in clients to the NED SED Service and the ASDC. Data may also be uploaded from a local file, a URL, a SAMP message from a VO-enabled tool, or through a custom file filter plug-in at run time.

portals as well as from outside resources like local files, URLs, other VO-enabled applications, and from plug-ins.

Iris natively supports IVOA-compliant FITS and VOTable formats (McDowell et al., 2012). Files in these formats will automatically be added to the user's workspace. The Builder can convert ASCII Tables, CSV, TSV, IPAC tables, and non IVOA-compliant VOTable and FITS files into the native format with user input. We provide two importing forms: (i) the SED importer, which handles spectrum-style files (i.e., those with columns for the spectral coordinate, flux/energy, and flux/energy uncertainties), and (ii) the Photometry Catalog Importer, which handles photometry catalogs (i.e., files where each column represents a passband and the cell values represent the corresponding fluxes, with an arbitrary number of rows). Users can save their setup options from the Import Setup Frame to a configuration file and automatically read-in files of the same format to Iris via the command line.

The SED Builder also has a hook for adding custom file filters. One could develop a custom file reader that would convert a non-standard file to an IVOA-compliant format. This kind of add-on would allow Iris to read non-standard files into Iris without requiring the use of the importer tools.

\subsubsection{Saving data}

Users can save entire SEDs or sets of Segments to IVOA-compliant VOTable or FITS files. In order to save all the metadata, the IVOA-compliant serializations rely on some specific constructs in the supported file formats, so that SEDs that have many different Segments can become very complicated to read for VO-unaware applications, although they retain all the metadata details. For instance, segments might have data expressed in different units inside the same SED.

To facilitate the ingestion of SEDs in VO-unaware applications and user scripts, we provide a simpler output format that only saves the minimum amount of meaningful information: the spectral coordinate, the flux or energy, and its uncertainties. As a result, the resulting SED file has only one Segment, with all the data expressed in a single set of units defined by the user. 
Whether the output includes all of the metadata or has a simplified single table format, the result is a compliant file that can be read back into Iris without any additional user's input.

This allows users to save a standardized version of the file that can be easily shared by Iris and by the user's scripts.

\subsection{NED SED Service}

Iris is packaged with a portal to the NED SED Service that, given a target name, retrieves all photometric data in NED associated with the source with that target name, and adds it to an existing SED.

In the context of the VAO development of Iris, we adapted the NED long standing photometry and spectral energy distribution service to conform as closely as practical to the relevant IVOA recommendations in order to deliver photometric data from the collection into Iris seamlessly. The objective for NED was to provide a working reference service for the development of Iris as well as to serve as a working prototype for new data protocols for spectrophotometric data being developed by the IVOA.

The NED SED Service returns data and information from the NED photometry collection (Mazzarella and NED Team, 2007). The NED SED Service provides three types of queries:

Information Discovery List objects with available photometry (SED) given a sky position (RA and Dec) and angular size. Also called a data discovery query.

Information Availability For a given named object, return the number of photometric data points.

Data Retrieval For a given named object, return the available photometric data in an IVOA Spectrum Data Model compatible VOTable.

All three query types use HTTP requests and responses which conform to the IVOA Simple Spectral Access Protocol Version 1.04 (SSAP; Tody et al., 2012); the responses are in VOTable format. The NED SED Service client in Iris employs the Data Retrieval query interface, and stores the response as a Segment. Photometric points with spectral line-based values and upper- and lower-limit values are excluded from the response.

Implementing a standard protocol interface, the NED SED service is also available through generic VO applications like TOPCAT and the VAO Data Discovery Too ${ }^{12}$.

\subsection{SED Viewer}

The Iris Viewer component is responsible for creating, managing, and providing user interactive feedback to spectral plots in Iris.

12 http://vao.stsci.edu/portal/Mashup/Clients/ Portal/DataDiscovery.html
The Viewer also provides most of the low-level GUI components used by the Fitting Tool component. The reason for this is that most, if not all of the GUI code used by both the Viewer and the Fitting Tool, were developed on top of the Specview (Busko, 2002, ascl:1210.016) code base.

Specview was developed in the late 1990's, initially as an experiment to evaluate Java graphics capabilities in the context of interactive spectral plotting. Over the years Specview grew from a simple visualizer dedicated mostly to plot spectral data from Hubble Space Telescope (HST) instruments, to a more capable tool with not only sophisticated visualization, but also data analysis capabilities. The ability to ingest spectral data from a variety of sources was also gradually incorporated into the tool, culminating with a Virtual Observatory interface capable of accessing services that comply with the SSAP standard.

Specview however kept the emphasis on spectral data, which is very different from the broad-band SED concept to which Iris is dedicated. Being initially conceived as a tool to support HST data, the design, and subsequent code implementation, were driven by the needs and requirements imposed by high-dispersion, relatively narrow-band spectra in the near-IR / optical / near-UV range. Thus some re-work was necessary to make Specview's internal data structures and algorithms comply with the data types associated with SEDs. Even so, a significant part of the code could be kept as is, thus realizing the savings associated with code re-use. This is particularly true in the case of the low-level graphics engine (Busko, 2000). Most of the work in adapting Specview's code base to Iris happened on two fronts: (i) adding code that implements the Iris Component interface, and (ii) augmenting the capabilities of the Data Browser to allow interactive access to SED metadata. Some work was also done in fine-tuning plotting capabilities to the particular needs of SED data.

The initial view the Viewer creates of a just-ingested SED is via a scatter plot depicting wavelengths (frequency and energy units are also supported) and flux density (or flux) for each data point that comprises the SED. The plot can be configured in a variety of ways, by changing the scaling and units. The data initially plotted can then be further examined in more detail, using tabular and tree depictions. In particular, the metadata associated with each data point, as well as the global metadata associated with the entire SED, can be examined in detail using the Metadata Browser. Data points can be selectively removed from the SED using filters sensitive to both data and metadata values. These filters are built by a userdefined Boolean expression that can be created and interacted with in the GUI itself. The expression uses Pythonlike syntax, and Python operators are available throughout. That way, SEDs can be modified after being read by the SED Builder, and before being further processed or measured. 


\subsection{Sherpa: Model Fitting}

Sherpa (ascl:1107.005) is the Chandra Interactive Analysis of Observations (CIAO; Fruscione et al., 2006, ascl:1311.006) modeling and fitting application. Sherpa enables the user to construct complex models from simple definitions and fit those models to $1 \mathrm{D}$ (spectra) and 2D (images) data using a variety of statistics and optimization methods.

Written in Python, with $\mathrm{C} / \mathrm{C}++/$ Fortran extensions, Sherpa was a robust choice for providing Iris with a curve fitting engine.

However, since the Iris front end was going to be a Java application ${ }^{13}$, an interoperability layer had to be designed to interface the graphical user interface and Sherpa as a fitting engine back-end.

SAMP is used as the interface protocol. This decision makes the design of the interface very simple, so that the interoperability layer on top of Sherpa is rather thin and consists only of the code required to inspect the incoming SAMP messages and build a call to Sherpa.

The design of this interface is represented schematically in Figure 8

When Iris is launched the sherpa-samp process is also started in the background. This process starts a SAMP client that waits for a SAMP hub to attach to, registering to a number of custom mtypes. The mtypes work as remote procedure identifiers, and SAMP messages provide the remote methods with data that need to be processed. Sherpa is used to compute a response that is packaged as a SAMP response to be shown to the user.

The thin layer between Java and Python code is implemented using two existing implementations of the SAMP protocol, namely jsamp ${ }^{14}$ for Java and SAMP y ${ }^{15}$ for Python.

The sherpa-samp layer grew to accommodate the new science requirements in the latest Iris releases, so to include some analysis code that is independent of Sherpa.

\subsubsection{Fitting Options}

We provide the following fitting optimization methods and fit statistics from Sherpa in Iris. Refsdal et al. (2009) discuss Sherpa's statistics in detail. Here, we briefly present the options.

The optimization methods available in Iris are variations of the Nelder-Mead simplex, Levenberg-Marquardt, and Monte Carlo algorithms. The Nelder-Mead simplex method, which finds the local minimum of a function in parameter space through a direct search method, is an adaptation of the algorithms described in Wright (1996) and

\footnotetext{
${ }^{13}$ In the first version of Iris the front end was a modified version of Specview itself, while in later versions we integrated different components under a common framework graphically represented by the Iris Desktop. Even in this configuration, the fitting front end was provided by Specview under the hood.

14 http://software.astrogrid.org/p/jsamp/1.3/

15 http://pythonhosted.org//sampy/
}

Lagarias et al. (1998). Levenberg-Marquardt optimization finds the local minimum of non-linear least squares functions of the model parameters (Moré, 1978). Lastly, the Monte Carlo method uses a differential evolution algorithm outlined in Storn and Price (1997) to find the global minimum in parameter space.

Sherpa provides several $\chi^{2}$ statistics with different variances. For example, users can use the variance of the $y$ uncertainties (or $y$-values if there are no uncertainties), or they can set the variance to 1 . Also included are two maximum likelihood functions based on Poisson statistics: Cash and C-statistic (Cash, 1979).

\subsection{Science Tools}

We provide built-in science tools that perform calculations commonly used in SED analysis: redshifting, interpolation, and integration. The data are setup on the Java-side of Iris, but the actual calculations are performed in sherpa-samp.

The open SEDs are listed in the Science Tools frame. The user selects the SED they wish to analyze, and inputs the required information for a calculation.

\subsubsection{Redshifting}

Redshifting SEDs in Iris refers to cosmological redshift. The spectral values are transformed into wavelength-space before shifting the SED. Because the apparent magnitude of a source is dimmer at high redshifts than low redshift, we correct the flux so that the area under the shifted SED equals that of the un-shifted SED using

$$
f_{z_{f}}(\lambda)=f_{z_{i}}(\lambda) \frac{\sum_{k=1}^{N}\left(f_{z_{i}}\left(\lambda_{k+1}\right)+f_{z_{i}}\left(\lambda_{k}\right)\right)}{\sum_{k=1}^{N}\left(f_{z_{f}}\left(\lambda_{k+1}\right)+f_{z_{f}}\left(\lambda_{k}\right)\right)},
$$

where $f_{z_{i}}$ is the observed flux at the initial (observed) redshift $z_{i}, f_{z_{f}}$ is the flux at the final (target) redshift $z_{f}, \lambda$ is the wavelength, $N$ is the number of points in the SED, and $\lambda_{k}$ is the wavelength of the $k^{t h}$ point in the SED. In sherpa-samp, we extend the astLib ${ }^{16}$ ast SED class that implements Equation 1 .

From the the user's perspective, the user supplies the initial and final redshift of the SED and clicks "Create New SED."

\subsubsection{Interpolation}

Iris provides 1D interpolation along the spectral axis. There are three interpolation options: linear, linear spline, and nearest neighbor. Interpolation may be carried out on a linear or logarithmic scale. Users may choose the number of bins, the spectral range over which to interpolate, and may choose to smooth the resultant SED via a boxcar method.

\footnotetext{
16 http: //astlib.sourceforge.net/
} 


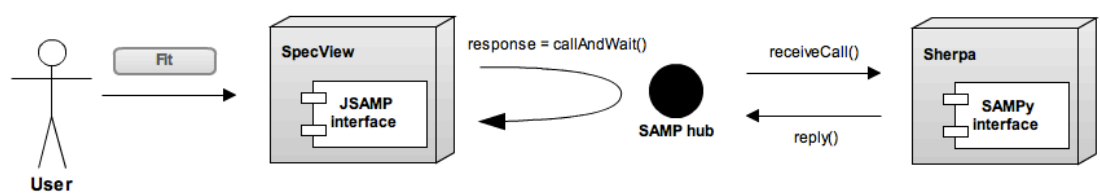

MType Protocol workflow

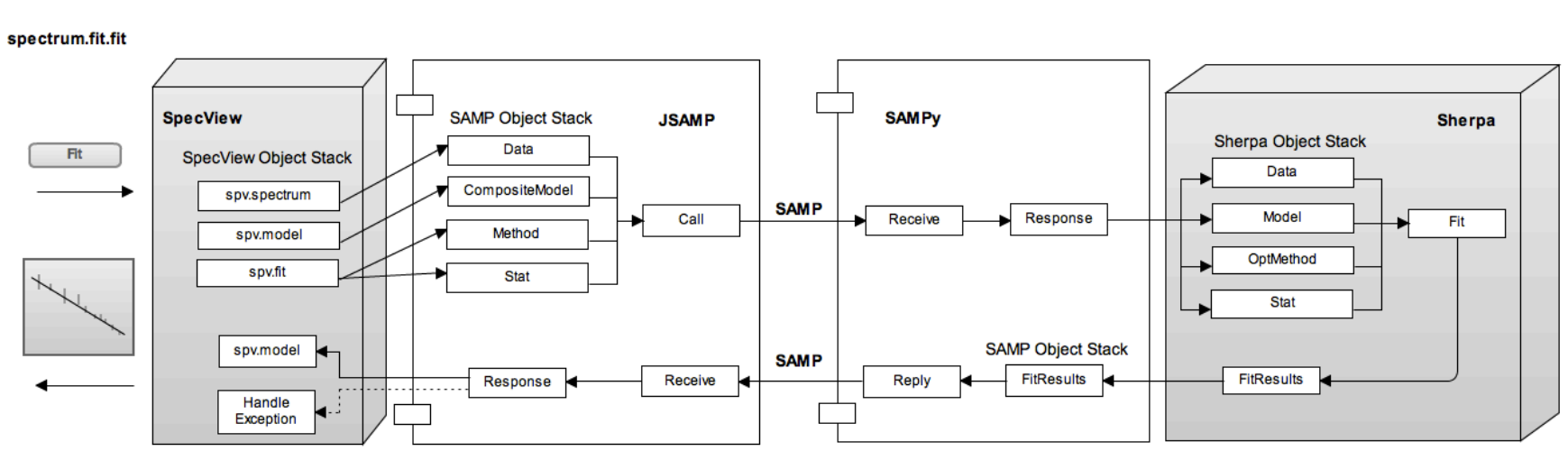

Figure 8: Design of the Specview/Sherpa interoperability layer. The interface between Sherpa (fitting engine), and Specview (graphical user interface) was designed by defining a common data model for representing the requests and responses of fitting operations: the serialization of the request is a SAMP message, whose mtype identifies the remote operation that the client is requesting.

\subsubsection{Integration}

The Integration tool was developed for estimating integrated fluxes of a SED. The tool acts as a wrapper of the astLib methods calcFlux and integrate, which in turn use the composite trapezoidal rule to integrate the SEDs.

Iris provides two methods of integration: (i) through a user-defined passband, and (ii) through a photometric filter. The first option lets the user specify the spectral range in wavelength, frequency, or energy units (Angstroms, Hz, and $\mathrm{keV}$, respectively) to integrate under. The second estimates the integrated flux measured through any of the photometric filters provided by the Spanish Virtual Observatory's (SVO's) Filter Profile Service ${ }^{17}$ (Solano, 2013). This service has an extensive collection of over 1000 filters at IR, optical, and UV instruments. The user chooses from a list of filters that can be searched by double-clicking on an instrument name, or by searching for a string in the browser. The user sees the minimum, maximum, and effective wavelengths of the filters before applying the filter to the SED. Both methods return the effective wavelength of the passband in Angstroms and the calculated flux in Jansky. The user can export the data to a new SED or save the results to a simple ASCII formatted file.

Notice that Iris currently integrates the SED data points, possibly after an interpolation, and not the model.

\footnotetext{
17 http://svo2.cab.inta-csic.es/theory/fps/index.php
}

So, if the transmission curves or passbands do not completely overlap with the SED Iris will return a NaN (Not a Number).

\section{Plug-ins: the Software Development Kit}

Iris offers a Java Software Development Kit (SDK) that can be used to extend the Iris capabilities through the use of dynamically pluggable add-ons, or plug-ins. The use cases for this are listed below.

New functionality A developer may want to add new capabilities in one or more new Components. This use case can be broken down in more detailed and concrete extensions, described later in this section.

Custom-to-Standard adapters A developer may want to create adapters that query a non-standard service, or load a non-standard dataset, and then turn the data to SEDLib objects, thus effectively standardizing them so that they can be used by other components in the Iris environment, or reused by other VO applications. In other terms, one can achieve interoperability using the Iris infrastructure starting from a non-interoperable service, file, or tool. Iris actually has some built-in Custom-to-Standard adapters, like the sherpa-samp layer described in Section 6, or the ASDC plug-in interface that queries a quasi-standard service, described in Section 7.2.2. 
This section describes Java plug-ins, while Section 3 described how users can extend the models for fitting SEDs using Python functions.

\subsection{Anatomy of a Plug-in}

A single Java Archive (jar) file can contain several plugins, and each plug-in can bundle several Iris Components.

Each Component can provide several additions to Iris, as described in some detail below.

\subsubsection{Menus and Buttons}

Usually, although not always, an Iris Component is visible to the user as either a set of buttons on the Iris Desktop, or as a set of menu items in the Iris menu bar, or both.

Menu items can be added to either the File menu or to the Tools menu in a specific plugin-related folder.

While the implementation of such buttons and menu items could be done from scratch by implementing some Java Interfaces, a set of abstract classes implements a lot of the boilerplate code and makes some convenient assumptions. This way buttons and menu items can be created with very few lines of code.

Menu items and buttons can be customized by providing the button name, a description that will be rendered as a mouse-hover tooltip, and icons.

\subsubsection{Command Line}

Iris offers a framework for providing simple command line interfaces to its tools. For example, Iris ships a command line interface to the SED Builder (see Section 6) that allows users to import non-standard files in bulk through scripts, possibly starting from templates saved interactively from the SED Builder.

The framework is extensible through a simple dispatching mechanism. Each component has a name that is used to dispatch the command line argument to the right CLI engine. For instance, the line

. Iris builder config.txt

instructs Iris to dispatch the config.txt argument to the SED Builder's CLI engine. Components bundled with plug-ins can provide such an engine by implementing the ICommandLineInterface Java Interface as shown in Listing 4 .

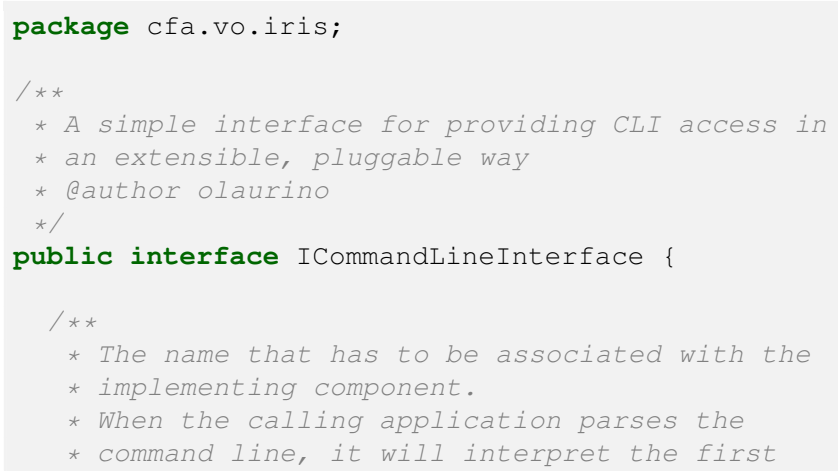

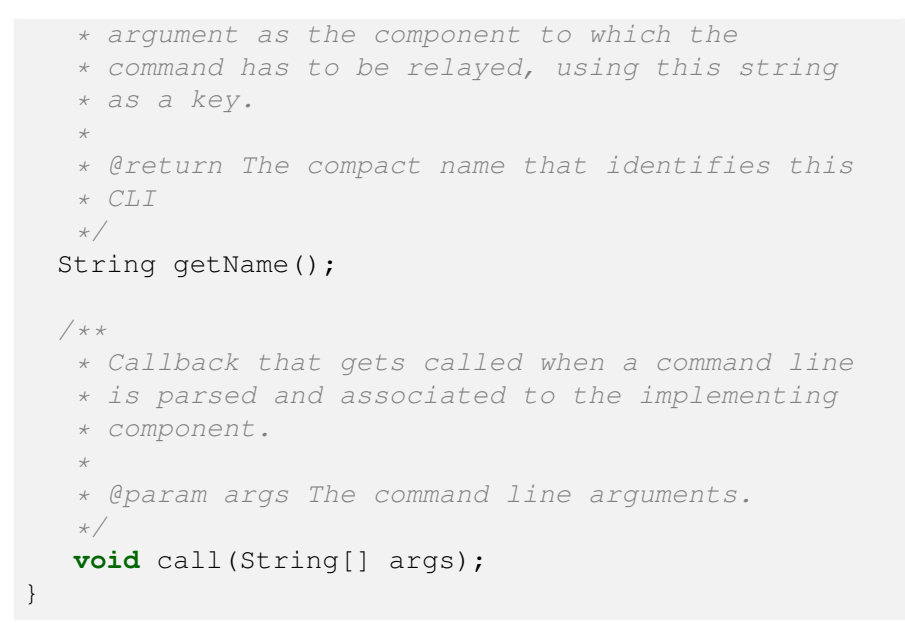

Listing 4: Every Iris component can expose a command line interface. Iris dispatches the command line arguments for the relative component to process. This code is written in Java.

\subsubsection{SAMP Handlers}

A possible extension that plug-ins can offer to the users is SAMP handlers. When Iris receives a SAMP message that matches the Handler's mtype, the message is directly dispatched to the Handler itself by the Iris framework. As a matter of fact, Iris just offers a convenient shortcut to the excellent jsamp implementation of SAMP, making it available to the users with just the bare minimum amount of work required. The setup of the SAMP infrastructure through jsamp is all done by Iris, including a keep-alive mechanism that brings a SAMP hub up when an existing one is shut down.

A hook is provided for Components willing to send their own SAMP messages to the SAMP Hub, again as a convenient shortcut to jsamp.

\subsubsection{Custom Events}

The Iris Events Framework is itself extensible: this way plug-in developers can, if needed, create their own nested architecture for their plug-in's Components.

\subsubsection{SED attachments}

Components can attach arbitrary objects to the SEDs managed by the SEDManager. This way users can rely on the Iris framework to manage the additional information they might want to store about the individual SEDs. When SEDs are deleted, the manager takes care of releasing any references to the attachments, reducing the risk of memory leaks.

\subsection{Plug-in examples}

\subsection{1. $A S D C$ - stable}

The Italian Space Agency Science Data Center (ASDC) hosts a database with tens of catalogs in a very wide range of wavelengths, also providing time domain information. 
A plug-in for providing Iris with a rich graphical user interface to query their database was developed by the ASDC in a collaboration between the ASDC and the Iris teams. The plug-in became part of the main Iris distribution in v2.0 and was a valuable test bench to review, validate, and improve the Iris Software Development Kit.

While the ASDC data query tool is now part of the Iris distribution, this tool provides a very good example of how a plug-in can be integrated seamlessly in the Iris framework to add specific value to the overall application. Integration can be so seamless, actually, that including the plug-in into the main Iris distribution is almost exclusively a matter of configuration rather than of coding.

The ASDC data query tool extends the capabilities of the SED Builder by providing a rich graphical user interface that allows users to check what archives to query, and since the ASDC query is a positional cone search, the client provides different adjustable search radii for each catalog that default to reasonable values consistent with the resolving power of the individual instruments.

Moreover, the tool allows users to query for specific observation time ranges, thus allowing basic time domain analysis of the SEDs.

This component proves several points about the Iris framework and SDK, as listed below.

Custom-to-Standard adapters The ASDC web service backing up the implementation of the query tool does not comply with any VO data access protocols (at least not yet), as this service was designed as a private interface to their database to be consumed by a dedicated client like the one implemented in Iris. The data files coming from the service, on the other hand, are compliant with the IVOA specifications, so they can be directly read by SEDLib and passed to the SEDManager.

Interoperability Although not designed as part of Iris, the ASDC plug-in integrates seamlessly with the Iris built-in components. When the ASDC query tool downloads data from the service, the data are listed in the SED Builder and visualized in the SED Viewer, even though the ASDC tool does not interact directly with any of them. They all interact only with the SED Manager and they get notified of changes by the events that are fired when Models are changed.

The Iris SDK As it will be explored in some detail in Section 7.4, a plug-in developer can pretty much focus on the implementation of the components' business logic, without worrying too much about the boiler plate code required to configure such components. By using the abstract classes that the Iris framework provides, one can leverage the existing components with just a few lines of code and then start adding value to the entire application.

\subsubsection{Vizier - experimental}

Experimental plug-ins are shipped with Iris but they can only be activated by turning on switches on the Iris command line. For instance, if one starts Iris with the command . / Iris --vizier an experimental plug-in 18 for the CDS Vizier photometric service gets loaded in the usual Iris desktop.

\subsection{3. $R$ - experimental}

A highly experimental proof-of-concept plug-in was developed to explore the possibility of interfacing Iris with rich analysis environments like $\mathrm{R}$. The plug-in shows how one can beam data from Iris to $\mathrm{R}$ and trigger some analysis on the dataset in $\mathrm{R} 19$

\subsection{Other Extensibility Points}

\subsubsection{Custom File Readers}

Iris supports a fair number of file formats natively: VOTable, FITS, CSV, TSV, ASCII, and IPAC tables. However, new file filters can be created and loaded at run-time. One can also create filters for the natively supported files. In this case, the custom filter would parse the file and map the metadata to the IVOA Data Model fields.

\subsubsection{Persistence}

Components can also get a handle to the configuration directory (usually a hidden folder in the user's home directory) if they need to persist information like user's preferences, local databases, or work sessions.

\subsection{How to write an Iris plug-in}

Iris uses Maven Archetypes to streamline the process of building and distributing Iris Java plug-ins.

You might also write plug-ins without using Maven, but you would need to take care of many steps that the Maven-generated project automatically takes care of, like the inclusion of your dependencies in your plug-in's jar file.

In order to have a test plug-in up and running you need to create a new project from the Maven archetype:

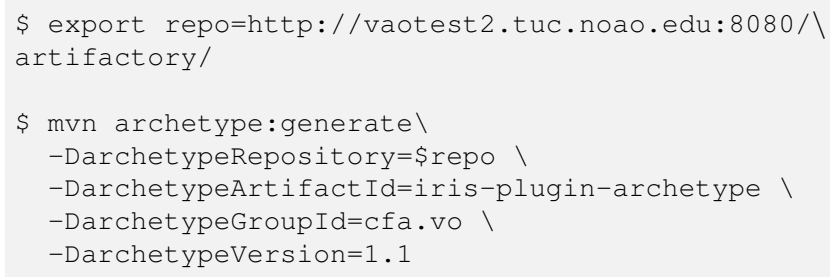

\footnotetext{
${ }^{18}$ While this client should work fine most of the time, users should not expect proper error handling, neither there is a way to change the search radius.

${ }^{19}$ In order to make this plug-in work one needs to install $\mathrm{R}$ and the Java- $\mathrm{R}$ interface package, and then set up some environment variables and start Iris in a non-standard way. If interested in experimenting with this plug-in, please contact the corresponding author.
} 
The above command will ask you some questions about the metadata for your plug-in project, like the group id, the project id (called artifact-id in Maven), and the version. At the end of the process you should have a directory named after your project-id. This directory contains all the files needed to build and package a test plug-in.

You can type mvn package from the newly created directory and Maven will package the test plug-in for you in the target directory as a jar file.

You can use the Iris Plug-in Manager component to install this jar file into Iris. As soon as the plug-in is installed, a new button should appear on the Iris desktop. If you click on the button, a rather impressive dialog box with the universal salutation "Hello World!" should appear on your screen.

You can inspect the source code of this project and notice that most of the code is made of metadata strings and basic class definitions and instantiations. By inheriting from the abstract classes that are provided with the Iris SDK, the actual code that one needs to implement starts from the implementation of the onclick callback of the AbstractPluginMenuItem class. From that call on, a plug-in developer can focus on the implementation of their components and start using the hooks provided by the Iris Framework in order to interoperate with the other Iris components, and possibly with other VO applications.

One can start from this dummy project, inspect the source code, make changes to the package and class names and to the metadata strings, and then start implementing their component's business logic and user interface.

The Iris website contains further documentation on how to write plug-ins, and you can contact the authors of this paper for further information.

\section{Future Plans}

We are working on improving Iris in several ways. With the VAO shutting down in 2014, the development of Iris has been taken over by the Chandra X-Ray Center group at the Smithsonian Astrophysical Observatory.

While the current Software Development Kit is focused on letting plug-ins contribute SEDs and SED segments to the user's workspace, we want to improve the ways in which plug-ins can interact with the visualization and fitting code, decoupling Specview and Sherpa.

We are also exploring solutions to overcome one of the limitations in the current code, namely the handling of high resolution spectra, that is mostly due to a visualization issue.

Several improvements will derive from the inclusion in Iris of the latest Sherpa version, and in particular of the new code for interpolating templates in template fitting. This will allow users to combine templates with other templates and functions and compute photometric redshifts through template fitting, for instance.
Also, we want to provide finer grained control over the visualization and manipulation of individual components in the model expressions.

From the user interface point of view, we are planning to provide Python bindings to enhance the integration of Iris in customized, complex scientific work-flows.

\section{Conclusions}

Iris is a Virtual Observatory application designed with the goal of streamlining the construction of broadband spectral energy distributions while providing flexible and robust tools for their analysis, with a stress on interoperability and extensibility.

To summarize, Iris provides: built-in capabilities for building, viewing, and analyzing broad-band spectro-photometric SEDs; a Python framework for fitting user-provided models and templates; interoperability with Virtual Observatory tools through the Simple Messaging Application Protocol (SAMP).

The Iris layered architecture takes advantage of the Virtual Observatory standards and protocols without exposing their complexity to the end users, who still benefit from the added interoperability. At the same time, developers can use a middle layer of abstraction that exposes the domain objects, i.e., photometric SEDs, and the user's workspace, in a clean and consistent way through a Java software development kit.

This way Iris combines several existing software components with new dedicated software, and provides hooks for astronomers and software developers that want to leverage the general interoperable framework while plugging in their own code.

Iris is available as an Open Source project, and can be downloaded as a binary or source distribution for Linux and OS X.

\section{Acknowledgments}

The Authors would like to acknowledge Giuseppina Fabbiano, Ian Evans, Jonathan McDowell, and Aneta Siemiginowska for their support and feedback in all the phases of the work. Dan Nguyen and Joseph Miller (SAO) supported the development team in the very early stages of the work.

We also thank the Italian Space Agency Science Data Center for the contribution of the ASDC Data plug-in, in particular Paolo Giommi, Roberto Primavera, Milvia Capalbi, and Bruce Gendre.

Support for the development of Iris was provided by the Virtual Astronomical Observatory contract AST0834235. Support for Sherpa is provided by the National Aeronautics and Space Administration through the Chandra X-ray Center, which is operated by the Smithsonian Astrophysical Observatory for and on behalf of the National Aeronautics and Space Administration contract NAS8-03060. 
Support for Specview is provided by the Space Telescope Science Institute, operated by the Association of Universities for Research in Astronomy, Inc., under National Aeronautics and Space Administration contract NAS5-26555. This research has made use of the NASA/IPAC Extragalactic Database (NED) which is operated by the Jet Propulsion Laboratory, California Institute of Technology, under contract with the National Aeronautics and Space Administration.

\section{References}

Acquaviva, V., Gawiser, E., Guaita, L., 2011. Spectral Energy Distribution Fitting with Markov Chain Monte Carlo: Methodology and Application to $\mathrm{z}=3.1 \mathrm{Ly} \alpha$-emitting Galaxies. ApJ 737, 47. doi $10.1088 / 0004-637 X / 737 / 2 / 47$.

Arnouts, S., Cristiani, S., Moscardini, L., Matarrese, S., Lucchin, F., Fontana, A., Giallongo, E., 1999. Measuring and modelling the redshift evolution of clustering: the Hubble Deep Field North. MNRAS 310, 540-556. doi 10.1046/j.1365-8711. $1999.02978 . \mathrm{x}$

Bayo, A., Rodrigo, C., Barrado Y Navascués, D., Solano, E., Gutiérrez, R., Morales-Calderón, M., Allard, F., 2008. VOSA: virtual observatory SED analyzer. An application to the Collinder 69 open cluster. A\&A 492, 277-287. doi 10.1051/0004-6361: 200810395

Benítez, N., 2000. Bayesian Photometric Redshift Estimation. ApJ 536, 571-583. doi $10.1086 / 308947$.

Berriman, G.B., Hanisch, R.J., Lazio, T.J.W., Szalay, A., Fabbiano, G., 2012. The organization and management of the Virtual Astronomical Observatory, in: Modeling, Systems Engineering, and Project Management for Astronomy V. doi 10.1117/12. 926605

Błażejowski, M., Siemiginowska, A., Sikora, M., Moderski, R., Bechtold, J., 2004. X-Ray Emission from the Quasar PKS 1127-145: Comptonized Infrared Photons on Parsec Scales. ApJLett 600, L27-L30. doi $10.1086 / 381497$

Bolzonella, M., Miralles, J.M., Pelló, R., 2000. Photometric redshifts based on standard SED fitting procedures. A\&A 363, 476-492. arXiv:astro-ph/0003380

Budavári, T., Wild, V., Szalay, A.S., Dobos, L., Yip, C.W., 2009. Reliable eigenspectra for new generation surveys. MNRAS 394, 1496-1502. doi 10.1111/j.1365-2966.2009.14415.x.

Busko, I., 2000. SPECVIEW: An Interactive Java Tool for Visualization and Analysis of Spectral Data, in: Astronomical Data Analysis Software and Systems IX, p. 79.

Busko, I., 2002. Specview: a Java Tool for Spectral Visualization and Model Fitting, in: Astronomical Data Analysis Software and Systems XI, p. 120.

Cash, W., 1979. Parameter estimation in astronomy through application of the likelihood ratio. ApJ 228, 939-947. doi 10.1086/ 156922

Chen, C.H., Jura, M., Gordon, K.D., Blaylock, M., 2005. A Spitzer Study of Dusty Disks in the Scorpius-Centaurus OB Association. ApJ 623, 493-501. doi $10.1086 / 428607$

Chiang, E.I., Goldreich, P., 1997. Spectral Energy Distributions of T Tauri Stars with Passive Circumstellar Disks. ApJ 490, 368-376. arXiv:astro-ph/9706042

Cid Fernandes, R., Gu, Q., Melnick, J., Terlevich, E., Terlevich, R., Kunth, D., Rodrigues Lacerda, R., Joguet, B., 2004. The star formation history of Seyfert 2 nuclei. MNRAS 355, 273-296. doi $10.1111 / j .1365-2966.2004 .08321 . x$

Conroy, C., 2013. Modeling the Panchromatic Spectral Energy Distributions of Galaxies. ARA\&A 51, 393-455. doi 10.1146/ annurev-astro-082812-141017

Czerny, B., Elvis, M., 1987. Constraints on quasar accretion disks from the optical/ultraviolet/soft X-ray big bump. ApJ 321, 305320. doi $10.1086 / 165630$
Dermer, C.D., Schlickeiser, R., 2002. Transformation Properties of External Radiation Fields, Energy-Loss Rates and Scattered Spectra, and a Model for Blazar Variability. ApJ 575, 667-686. doi $10.1086 / 341431$.

Doe, S., et al., 2012. Iris: The VAO SED Application, in: Astronomical Data Analysis Software and Systems XXI, p. 893. arXiv:1205.2419.

Evans, J.D., et al., 2012. Managing distributed software development in the Virtual Astronomical Observatory, in: Modeling, Systems Engineering, and Project Management for Astronomy V. doi 10 . $1117 / 12.927371$

Freeman, P., Doe, S., Siemiginowska, A., 2001. Sherpa: a missionindependent data analysis application, in: Astronomical Data Analysis, pp. 76-87. doi $10.1117 / 12.447161$.

Fruscione, A., et al., 2006. CIAO: Chandra's data analysis system, in: Observatory Operations: Strategies, Processes, and Systems. doi $10.1117 / 12.671760$

Ilbert, O., et al., 2006. Accurate photometric redshifts for the CFHT legacy survey calibrated using the VIMOS VLT deep survey. A\&A 457, 841-856. doi 10.1051/0004-6361:20065138.

Johnson, R.E., Foote, B., 1988. Designing Reusable Classes. Journal of Object-Oriented Programming 1, 22-35. http://www. laputan.org/drc.html.

Lagarias, J.C., Reeds, J.A., Wright, M.H., Wright, P.E., 1998. Convergence Properties of the Nelder-Mead Simplex Method in Low Dimensions. SIAM J. on Optimization 9, 112-147. doi $10.1137 /$ S1052623496303470

Lagrange, A.M., Backman, D.E., Artymowicz, P., 2000. Planetary Material around Main-Sequence Stars. Protostars and Planets IV , 639 .

Laurino, O., Busko, I., Cresitello-Dittmar, M., D’Abrusco, R., Doe, S., Evans, J., Pevunova, O., Norris, P., 2013. Constructing and Analyzing Spectral Energy Distributions with the Virtual Observatory, in: American Astronomical Society Meeting Abstracts, p. 240.38 .

Massaro, E., Tramacere, A., Perri, M., Giommi, P., Tosti, G., 2006. Log-parabolic spectra and particle acceleration in blazars. III. SSC emission in the $\mathrm{TeV}$ band from Mkn501. A\&A 448, 861-871. doi 10.1051/0004-6361:20053644.

Mazzarella, J.M., NED Team, 2007. NED for a New Era, in: Astronomical Data Analysis Software and Systems XVI, p. 153.

McDowell, J., et al., 2012. IVOA Recommendation: Spectrum Data Model 1.1. ArXiv e-prints arXiv:1204.3055.

Moré, J.J., 1978. The levenberg-marquardt algorithm: Implementation and theory, in: Watson, G. (Ed.), Numerical Analysis. Springer Berlin Heidelberg. volume 630 of Lecture Notes in Mathematics, pp. 105-116. doi $10.1007 /$ BFb0067700

Ochsenbein, F., et al., 2011. IVOA Recommendation: VOTable Format Definition Version 1.2. ArXiv e-prints arXiv:1110.0524.

Quinn, P.J., et al., 2004. The International Virtual Observatory Alliance: recent technical developments and the road ahead, in: Optimizing Scientific Return for Astronomy through Information Technologies, pp. 137-145. doi $10.1117 / 12.551247$

Refsdal, B.L., et al., 2009. Sherpa: 1D/2D modeling and fitting in Python, in: Proceedings of the 8th Python in Science Conference, Pasadena, CA, 2009, edited by G. Varoquaux, S. van der Walt and J. Millman, p. 51.

Robitaille, T.P., Whitney, B.A., Indebetouw, R., Wood, K., 2007. Interpreting Spectral Energy Distributions from Young Stellar Objects. II. Fitting Observed SEDs Using a Large Grid of Precomputed Models. ApJS 169, 328-352. doi 10.1086/512039

Robitaille, T.P., Whitney, B.A., Indebetouw, R., Wood, K., Denzmore, P., 2006. Interpreting Spectral Energy Distributions from Young Stellar Objects. I. A Grid of 200,000 YSO Model SEDs. ApJS 167, 256-285. doi $10.1086 / 508424$

Sawicki, M., Yee, H.K.C., 1998. Optical-Infrared Spectral Energy Distributions of Z ¿ 2 Lyman Break Galaxies. AJ 115, 1329-1339. doi $10.1086 / 300291$.

Shapley, A., Fabbiano, G., Eskridge, P.B., 2001. A Multivariate Statistical Analysis of Spiral Galaxy Luminosities. I. Data and Results. ApJS 137, 139-199. doi 10.1086/322998 
Smith, J.D.T., et al., 2007. The Mid-Infrared Spectrum of Starforming Galaxies: Global Properties of Polycyclic Aromatic Hydrocarbon Emission. ApJ 656, 770-791. doi 10.1086/510549

Solano, E., 2013. Spectral stellar libraries and the Virtual Observatory. ArXiv e-prints arXiv: 1312.3249

Storn, R., Price, K., 1997. Differential evolution \&ndash; a simple and efficient heuristic for global optimization over continuous spaces. J. of Global Optimization 11, 341-359. doi 10.1023/A: 1008202821328

Taylor, M., Boch, T., Fitzpatrick, M., Allan, A., Paioro, L., Taylor, J., Tody, D., 2011. IVOA Recommendation: SAMP - Simple Application Messaging Protocol Version 1.3. ArXiv e-prints arXiv: 1110.0528

Taylor, M.B., 2005. TOPCAT \& STIL: Starlink Table/VOTable Processing Software, in: Astronomical Data Analysis Software and Systems XIV, p. 29.

Tody, D., et al., 2012. IVOA Recommendation: Simple Spectral Access Protocol Version 1.1. ArXiv e-prints arXiv:1203.5725.

Tramacere, A., Giommi, P., Perri, M., Verrecchia, F., Tosti, G., 2009. Swift observations of the very intense flaring activity of Mrk 421 during 2006. I. Phenomenological picture of electron acceleration and predictions for $\mathrm{MeV} / \mathrm{GeV}$ emission. A\&A 501, 879-898. doi $10.1051 / 0004-6361 / 200810865$

Vrtilek, S.D., Raymond, J.C., Garcia, M.R., Verbunt, F., Hasinger, G., Kurster, M., 1990. Observations of Cygnus X-2 with IUE Ultraviolet results from a multiwavelength campaign. A\&A 235, 162-173.

Walcher, J., Groves, B., Budavári, T., Dale, D., 2011. Fitting the integrated spectral energy distributions of galaxies. Ap\&SS 331, 1-52. doi 10.1007/s10509-010-0458-z

Wright, M.H., 1996. Direct Search Methods: Once Scorned, Now Respectable, in: Griffiths, D.F., Watson, G.A. (Eds.), Numerical Analysis 1995 (Proceedings of the 1995 Dundee Biennial Conference in Numerical Analysis), CRC Press. pp. 191-208. 